\title{
What is the best adjustment of appendicular lean mass for predicting mortality or disability among Japanese community dwellers?
}

Rei Otsuka ${ }^{1 *}$ (D, Yasumoto Matsui ${ }^{2}$, Chikako Tange ${ }^{1}$, Yukiko Nishita ${ }^{1}$, Makiko Tomida ${ }^{1}$, Fujiko Ando ${ }^{3}$, Hiroshi Shimokata ${ }^{4}$ and Hidenori Arai $^{5}$

\begin{abstract}
Background: Age-related declines in skeletal muscle mass and strength, representing "sarcopenia," are a growing concern in aging societies. However, the prevalence of low muscle mass based on the height ${ }^{2}$-adjustment has been shown to be extremely low, and a more appropriate definition of low muscle mass is needed, particularly for Asian women. The aim of this study was to explore the most appropriate adjustment of appendicular lean mass (ALM) for predicting mortality or disability risk using ALM or any of 5 adjustments of ALM among communitydwelling Japanese.
\end{abstract}

Methods: Subjects comprised 1026 men and 952 women between 40 and 79 years old at baseline (1997-2000) who participated in the National Institute for Longevity Sciences - Longitudinal Study of Aging, Japan. ALM (kg) and 5 adjusted indices of ALM (ALM/leg length, ALM/height, ALM/height ${ }^{2}$, ALM/weight, and ALM/body mass index $[\mathrm{BMI}]$ ) were assessed at baseline. Disability was defined by long-term care insurance certification based on responses to a survey mailed in 2013, and death records were obtained as vital statistics until December 2014. Crude and adjusted Cox proportional hazard models were used to estimate hazard ratios for mortality or disability by sex-stratified quintiles of each ALM index (ALM and adjusted ALM) or sarcopenia-related indices. The area under the curve (AUC) was calculated with the multivariate-adjusted logistic regression model. Additionally, mixed-effects analyses were used to clarify the age-related ALM indices decline over 12 years $(n=1838)$.

Results: Crude Cox proportional hazard models and multivariate-adjusted logistic model (AUC) indicated that higher ALM and ALM/BMI in women, and higher ALM, ALM/leg length, ALM/height, and ALM/BMI in men were associated with lower risks for mortality or disability than ALM/height ${ }^{2}$. The mixed effect model indicated all ALM indices in men, and ALM, ALM/leg length, and ALM/height in women could better predict age-related lean muscle mass decline.

Conclusions: Unadjusted ALM in women, and ALM/leg length, ALM/height, ALM/BMI, and ALM in men may be more appropriate for predicting future mortality or disability than ALM/height ${ }^{2}$. Considering the age-related muscle mass decline, unadjusted ALM would be the first variable to assess, regardless of sex, in this Japanese cohort study.

Keywords: Japanese, Skeletal muscle mass, Sarcopenia, Criteria, Mortality, Disability

\footnotetext{
* Correspondence: otsuka@ncgg.go.jp

${ }^{1}$ Section of NILS-LSA (National Institute for Longevity Sciences-Longitudinal

Study of Aging), Center for Gerontology and Social Science, National Center

for Geriatrics and Gerontology, 7-430 Morioka-cho, Obu, Aichi 474-8511,

Japan

Full list of author information is available at the end of the article
} 


\section{Background}

Age-related declines in skeletal muscle mass and strength, referred to as "sarcopenia" [1-3], are a growing concern in Asian countries as the population ages [4], because sarcopenia is associated with adverse health outcomes in older adults [5]. In 2014, the Asian Working Group for Sarcopenia (AWGS) announced the development of a diagnostic algorithm for sarcopenia for Asians [6]. The AWGS recommended using height ${ }^{2}$-adjusted skeletal muscle mass index (SMI) values rather than weight-adjusted SMI values $[2,7]$. However, the prevalence of low muscle mass based on the height ${ }^{2}$-adjustment among Asian women was shown to be extremely low [8], and height ${ }^{2}$-adjusted muscle mass was not shown to decrease with age $[9,10]$.

The Foundation for the National Institutes of Health (FNIH) Sarcopenia Project proposed "appendicular lean mass (ALM) adjusted body mass index (BMI)" as cut-off points for low lean mass in men and women in 2014 [11]. The sarcopenia criteria using these definitions by FNIH better predicted mortality among Korean men, and there was no positive association in women [12]. However, using the lowest quintile in that study showed better predictive value to estimate mortality in women than using ALM/height ${ }^{2}$. An alternative and more appropriate definition of low muscle mass is still needed [13], particularly for Asian women.

The research question in this study was "what is the best adjustment of appendicular lean mass for predicting mortality or disability among Japanese community dwellers?" To explore the most appropriate skeletal muscle mass adjustment in Asians, we measured total ALM and used 5 different adjustments-ALM/leg length, ALM/height, ALM/height ${ }^{2}$, ALM/weight, and ALM/body mass index (BMI) - to examine the associations of ALM and each adjustment with mortality or disability among communitydwelling Japanese individuals. Four of 5 of these skeletal muscle mass adjustments were chosen according to previous studies [14-16], and we added ALM/leg length adjustment as a fifth variable. We hypothesized leg length for ALM adjustment would better predict age-related ALM decline than using height adjustments because we considered adjustments using height ${ }^{2}$ might result in over-adjustment for the elderly, as upper body height, especially in women, tends to shorten with age.

\section{Methods}

\section{Study cohort}

Data were collected as part of the National Institute for Longevity Sciences-Longitudinal Study of Aging (NILS-LSA). In this project, the normal aging process has been assessed over time using detailed questionnaires, medical check-ups, anthropometric measurements, physical fitness tests, and nutritional examinations. Participants in the NILS-LSA included randomly selected age- and sex- stratified individuals from the non-institutionalized residents in the institute neighbourhood areas of Obu City and Higashiura Town in Aichi Prefecture in Japan. The first wave of the NILS-LSA was conducted from 1997 to 2000 and included 2267 participants (1139 men, 1128 women; age range, 40-79 years). Details of the NILSLSA study have been reported elsewhere [17]. Subjects were followed-up every 2 years from the second to seventh wave (2000-2012).

\section{Follow-up survey and vital statistics records}

In July 2013, a self-administered questionnaire was mailed to participants to assess health status, including a "requirement for long-term care" under the new long-term care insurance system that started in Japan in 2000 [18, 19]. In addition, we obtained death records for all participants and obtained information from local government regarding which participants had moved to other areas. To clarify causes of death, we used National Vital Statistics records that were available until the end of December 2014. Mortality or disability was defined according to the National Vital Statistics records, or self-reported long-term care insurance certification, respectively. The main outcome in this study was "composite outcome for mortality or disability" as we combined these outcomes to increase the statistical power (to increase the number of cases).

\section{Study subjects}

Among the 2267 participants who participated in the first wave, we excluded 289 patients with a history of Parkinson's disease $(n=5)$ or for whom data were missing $(n=284)$ (Fig. 1). Of the 1978 participants still being followed as of 2014,389 men and women were categorized as having died ( $n=299)$ or as needing long-term care insurance certification $(n=90)$. Of the 1589 men and women categorized as "censored," 1481 were confirmed as alive according to information from the local government, and 108 had moved away from the local area or dropped out.

The study protocol was reviewed and approved by the Committee of Ethics of Human Research at the National Center for Geriatrics and Gerontology. Written informed consent was obtained from all subjects during the first to the seventh wave of the NILS-LSA. In the survey mailed in 2013, we explained that returning the self-administered questionnaire represented informed consent. Death data were obtained by means of secondary usage of demographic statistics by predetermined procedures. We used anonymous death data that had already been collected by the Ministry of Health, Labour and Welfare in Japan and do not require consent of individuals.

Assessment of muscle mass ALM $(\mathrm{kg})$, which represents the appendicular fat-free mass minus the bone mineral content [10], was assessed using a QDR-4500 


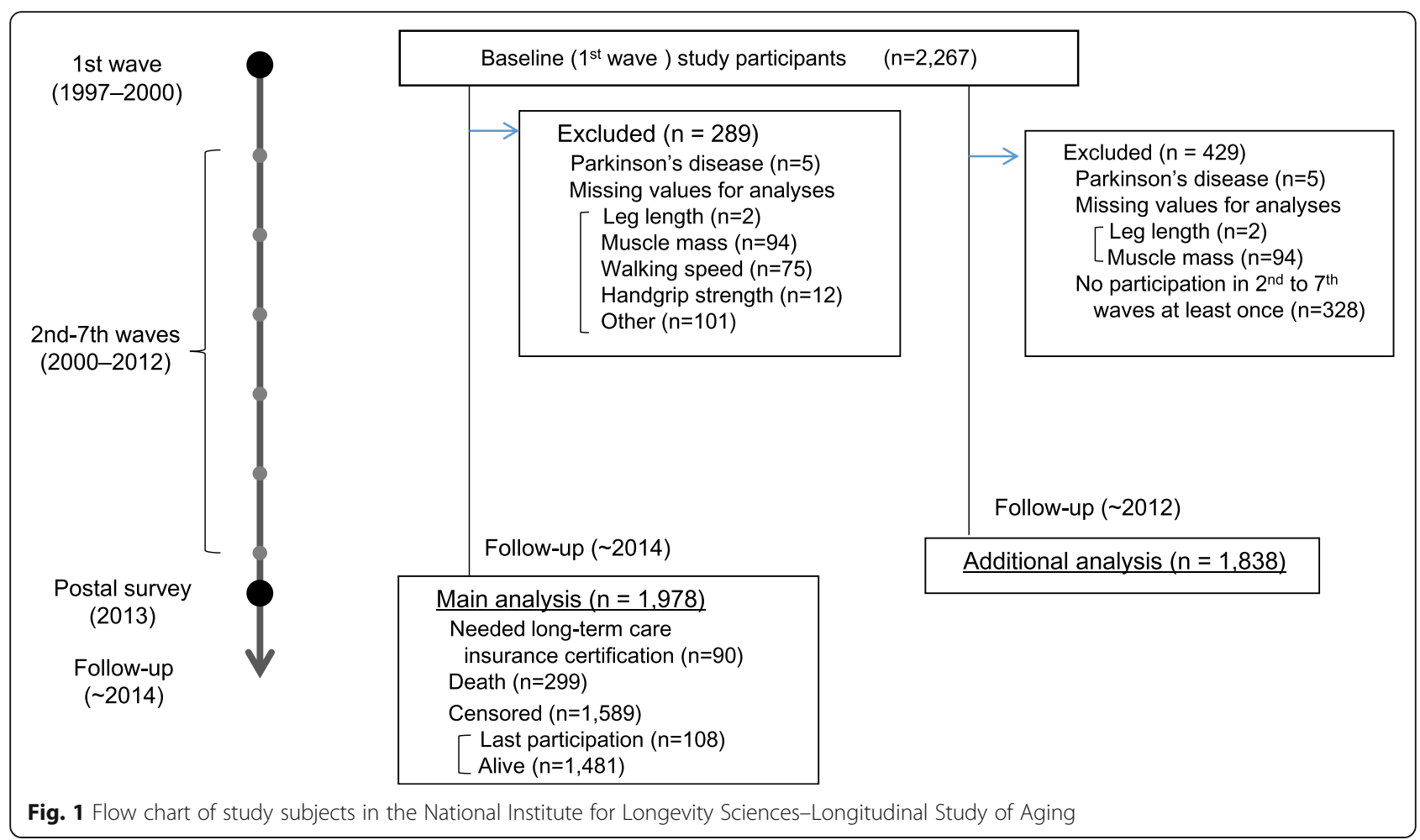

dual-energy X-ray absorptiometry (DXA) system (Hologic, Bedford, MA).

We calculated 5 indices using ALM (kg): ALM divided by leg length $(\mathrm{kg} / \mathrm{m})$; ALM divided by height $(\mathrm{kg} / \mathrm{m})$; ALM divided by height squared $\left(\mathrm{kg} / \mathrm{m}^{2}\right)$; ALM divided by weight and multiplied by $100\left(\mathrm{~kg} / \mathrm{kg}^{*} 100\right)$; and ALM divided by BMI and multiplied by $10\left(\mathrm{~kg} / \mathrm{kg} / \mathrm{m}^{2 *} 10\right)$. BMI was calculated as weight divided by height squared.

\section{Other measurements}

History of stroke, hypertension, hyperlipidemia, heart disease, and diabetes (past and current), current smoking status (yes/no), education ( $\leq 9$ or $\geq 10$ years of school), and annual family income $(<5,500,000$ or $\geq 5,500,000$ yen per year) were collected using self-reported questionnaires, and medical doctors or trained staff confirmed the information [20]. Sarcopenia was defined according to AWGS [6] criteria using grip strength, gait speed, and muscle mass. All measurements were assessed in the first-wave survey of the NILS-LSA.

\section{Statistical analysis}

Sex differences in baseline characteristics of participants were analysed using the $t$ test or $x^{2}$ test. The difference in the prevalence of mortality or disability by sex-stratified quintiles of each ALM index (ALM and adjusted ALMs) or sarcopenia-related indices was analysed using the $x^{2}$ test. Crude and adjusted Cox proportional hazard models were used to estimate hazard ratios (HRs) and 95\% confidence intervals (CIs) for mortality or disability by sex-stratified quintiles of each ALM index (ALM and adjusted ALMs) or sarcopenia-related indices. For Cox models, follow-up time (years) was calculated by the duration (days) that had elapsed since the day on which each participant entered the first wave of the NILS-LSA. The last day of follow-up for each participant was used for analysis, as either the date of death, the earliest day of needing long-term care (event group), the latest day of last participation in the NILS-LSA, or December 2014, whichever came first (censored group). When the participants were missing, that is, they moved away from the local area or dropped out $(n=108)$, we considered the last participation day of the NILS-LSA as the last day of follow-up.

Variables considered for adjustment were age, smoking status, education, family income, history of stroke, hypertension, heart disease, hyperlipidemia, and diabetes mellitus (multivariate-adjusted). In sub-analyses, we calculated receiver-operating-characteristic (ROC) curves on disability or mortality according to the sexstratified quintiles of each ALM index (ALM and adjusted ALMs). The area under the ROC curve (AUC) was calculated with the multivariate-adjusted logistic regression model.

Probability levels of $<.05$ and $<.10$ were considered significant and marginally significant, respectively. All statistical analyses were conducted using Statistical Analysis System software version 9.3 (SAS Institute, Cary, NC). 


\section{Additional analyses}

To clarify age-related changes in each ALM index (ALM alone and ALM with each of the 5 adjustments), we attempted to describe trends in changes to these indices over 12 years according to age in the first wave of the study of the NILS-LSA.

Among the 2267 participants (age range, $40-79$ years) who participated in the first wave, we excluded patients with a history of Parkinson's disease $(n=5)$ or for whom data were missing $(n=96)$ (Fig. 1). We selected subjects who also participated in more than one study wave from the second to the seventh wave, because variables could be followed-up at least once from the first wave. A total of 1838 subjects ( 951 men, 887 women) who were in the first wave were available for analysis. Each wave was conducted every 2 years. Mean (SD) interval and participation times between the first and last waves of participation for each participant were 5.5 (4.1) years and 3.7 (2.0) times, respectively.

For repeated-measures analyses of each ALM index, a mixed-effects model was used. To estimate fixed effects on each ALM index by follow-up time, both age at baseline and the interaction of follow-up time $x$ age were substituted into the model. To clarify the impact of habitual lifestyles on each ALM index, we additionally adjusted the mixed effect model using lifestyle-related factors including smoking status, alcohol intake, total physical activity, and energy intake.

\section{Results}

Baseline characteristics of study participants are shown in Table 1. Mean age was 58.8 years in men and 58.5 years in women. Mean BMI was 23.0 in men and 22.8 in women. Significantly more men had diabetes, and significantly more women had dyslipidemia. Mean ALM index (ALM and adjusted ALMs) were significantly higher in men than in women.

Tables 2 and 3 show the associations between baseline ALM indices and composite outcomes of mortality or disability in men and women, respectively. Before the Cox proportional hazard models, we tested the difference of the prevalence of mortality or disability by sex-stratified quintiles of each ALM index (ALM and adjusted ALMs) or sarcopenia-related indices. In men, the prevalence of mortality or disability was different according to the quintiles of all ALM indexes. In women, the prevalence was statistically different in ALM, ALM/leg length, and ALM/BMI only.

The coincidence rate of the lowest quintile among each ALM index (ALM and adjusted ALMs) are shown in Additional file 1: Table S1. In men and women, there were higher coincident rates (more than $80 \%$ ) between ALM and ALM/leg length, ALM/height indexes, or ALM/ leg length and ALM/height indexes, or ALM/height and ALM/height ${ }^{2}$ indexes, respectively.
Table 1 Baseline characteristics of study participants

\begin{tabular}{|c|c|c|c|}
\hline Characteristic & $\begin{array}{l}\text { Men } \\
(n=1026)\end{array}$ & $\begin{array}{l}\text { Women } \\
(n=952)\end{array}$ & $P^{*}$ \\
\hline Age, years, mean (SD) & $58.8(10.9)$ & $58.5(10.8)$ & .588 \\
\hline Weight, kg, mean (SD) & $62.4(9.2)$ & $52.6(8.0)$ & $<.001$ \\
\hline Height, cm, mean (SD) & $164.7(6.4)$ & $151.7(5.9)$ & $<.001$ \\
\hline Leg length, cm, mean (SD) & $80.3(4.1)$ & $74.2(3.6)$ & $<.001$ \\
\hline $\mathrm{BMI}, \mathrm{kg} / \mathrm{m}^{2}$, mean (SD) & $23.0(2.8)$ & $22.8(3.1)$ & .455 \\
\hline ALM, kg, mean (SD) & $20.1(2.8)$ & $14.1(2.0)$ & $<.001$ \\
\hline ALM/leg length, kg/m, mean (SD) & $25.0(3.1)$ & $18.9(18.8)$ & $<.001$ \\
\hline ALM/height, kg/m, mean (SD) & $12.2(1.4)$ & $9.3(1.1)$ & $<.001$ \\
\hline ALM/(height) ${ }^{2}, \mathrm{~kg} / \mathrm{m}^{2}$, mean (SD) & $7.3(5.0)$ & $6.1(4.2)$ & $<.001$ \\
\hline ALM/weight, $\mathrm{kg} / \mathrm{kg} \times 100$, mean (SD) & $32.4(2.3)$ & $26.7(2.3)$ & $<.001$ \\
\hline ALM/BMI, kg/kg/m²×10, mean (SD) & $8.8(1.0)$ & $6.2(0.8)$ & $<.001$ \\
\hline Current smoker, n (\%) & $388(37.8)$ & $69(7.2)$ & $<.001$ \\
\hline Education, $\leq 9$ years, $n(\%)$ & $297(28.9)$ & $342(35.9)$ & $<.001$ \\
\hline $\begin{array}{l}\text { Family income, }<5,500,000 \\
\text { yen/year, } n(\%)\end{array}$ & $395(38.5)$ & $429(45.1)$ & .003 \\
\hline Stroke, $n(\%)$ & $34(3.3)$ & $13(1.4)$ & .005 \\
\hline Hypertension, n (\%) & $236(23.0)$ & $241(25.3)$ & .230 \\
\hline Heart disease, $n(\%)$ & $121(11.8)$ & $97(10.2)$ & .255 \\
\hline Hyperlipidemia, n (\%) & $135(13.2)$ & $187(19.6)$ & $<.001$ \\
\hline Diabetes, $n$ (\%) & $102(9.9)$ & $45(4.7)$ & $<.001$ \\
\hline
\end{tabular}

$S D$ standard deviation, $B M I$ body mass index, $A L M$ appendicular lean mass "The $t$-test was used for continuous variables, and the $x^{2}$ test was used for categorical variables

We divided the cohort into quintiles and determined HRs in crude, age-adjusted and multivariate-adjusted models. Men with higher ALM, ALM/leg length, ALM/ height, and ALM/BMI values displayed a lower risk for mortality or disability (crude), although these relationships disappeared after adjusting for age. In the multivariateadjusted model, none of the ALM indices was positively associated with outcomes. Women with high ALM and higher ALM/BMI values displayed a lower risk for mortality or disability (crude), although these relationships again disappeared after adjusting for age. In the multivariateadjusted model, none of the ALM indices was positively associated with outcomes. The AUC was the highest in the ALM/leg length followed by ALM/height in men. In women, the AUC was the highest in the ALM/BMI followed by ALM.

Additional file 2: Table S2 shows mixed-effects analyses of the fixed effects of ALM indices over 12 years. The fixed effect of age and the interaction of age and time on ALM and the 5 ALM adjustments were statistically significant in men. In women, both the fixed effect of age and the interaction of age and time were statistically significant for ALM, ALM/leg length, ALM/height, and ALM/weight. Slopes of the ALM and 5 ALM adjustments in men and 


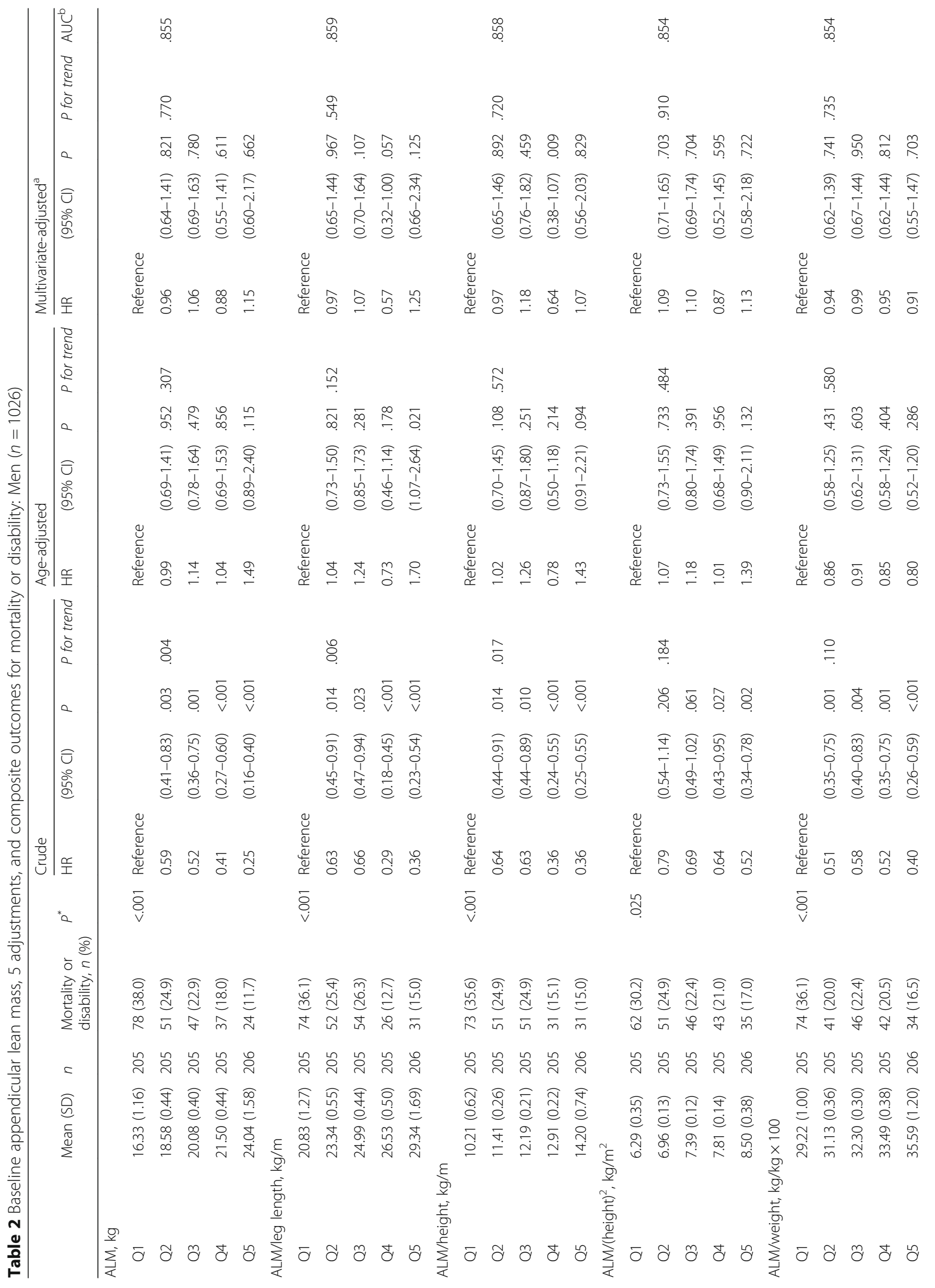




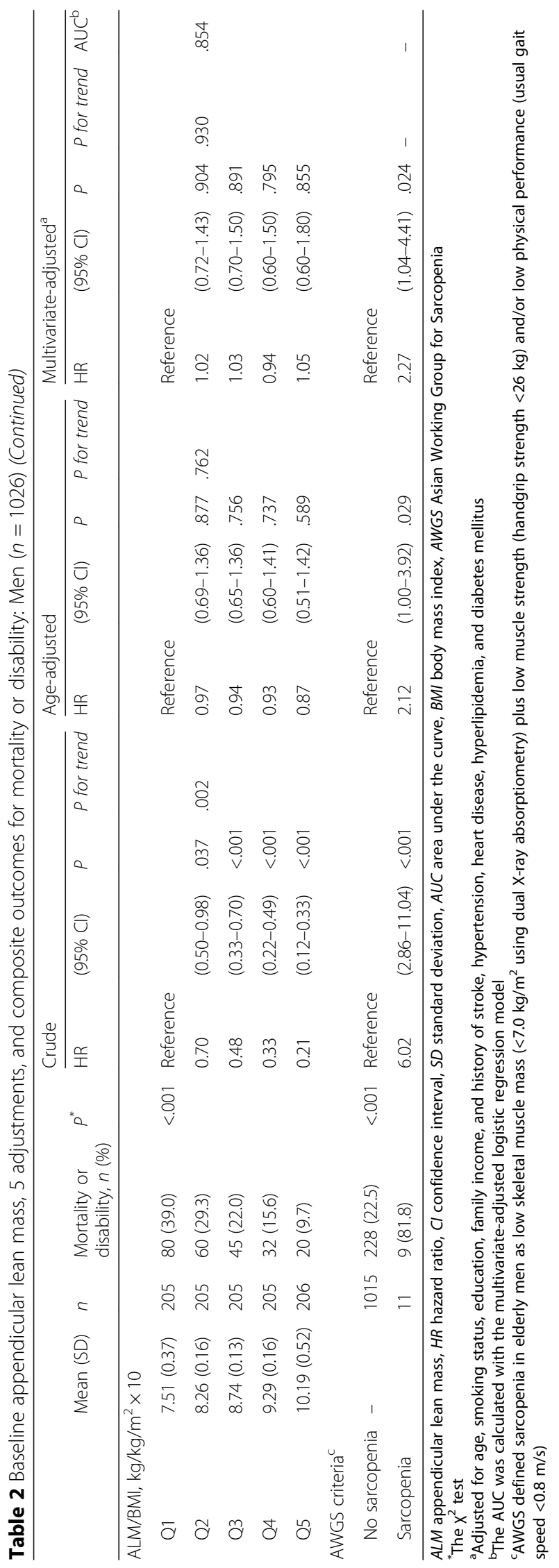




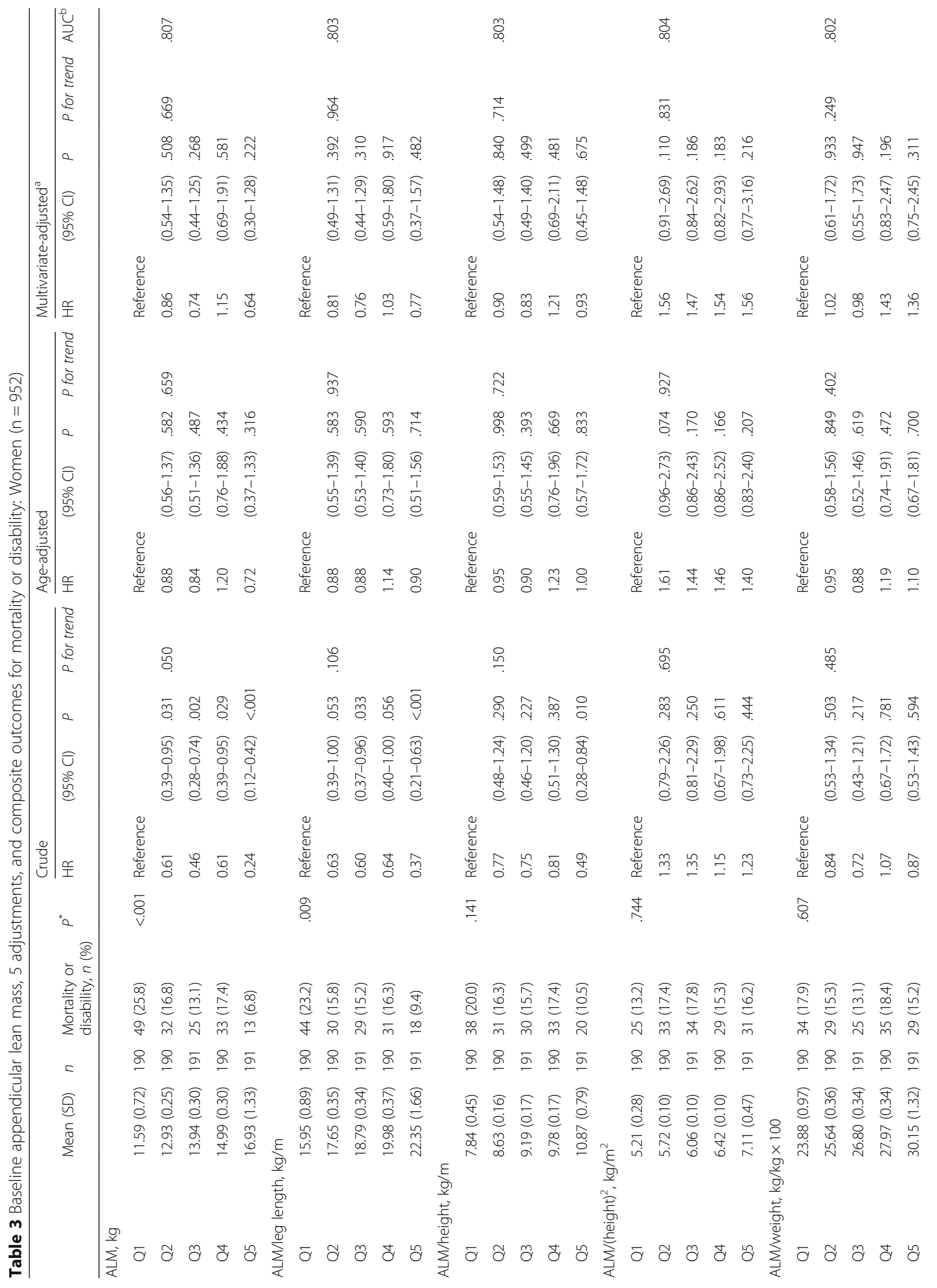




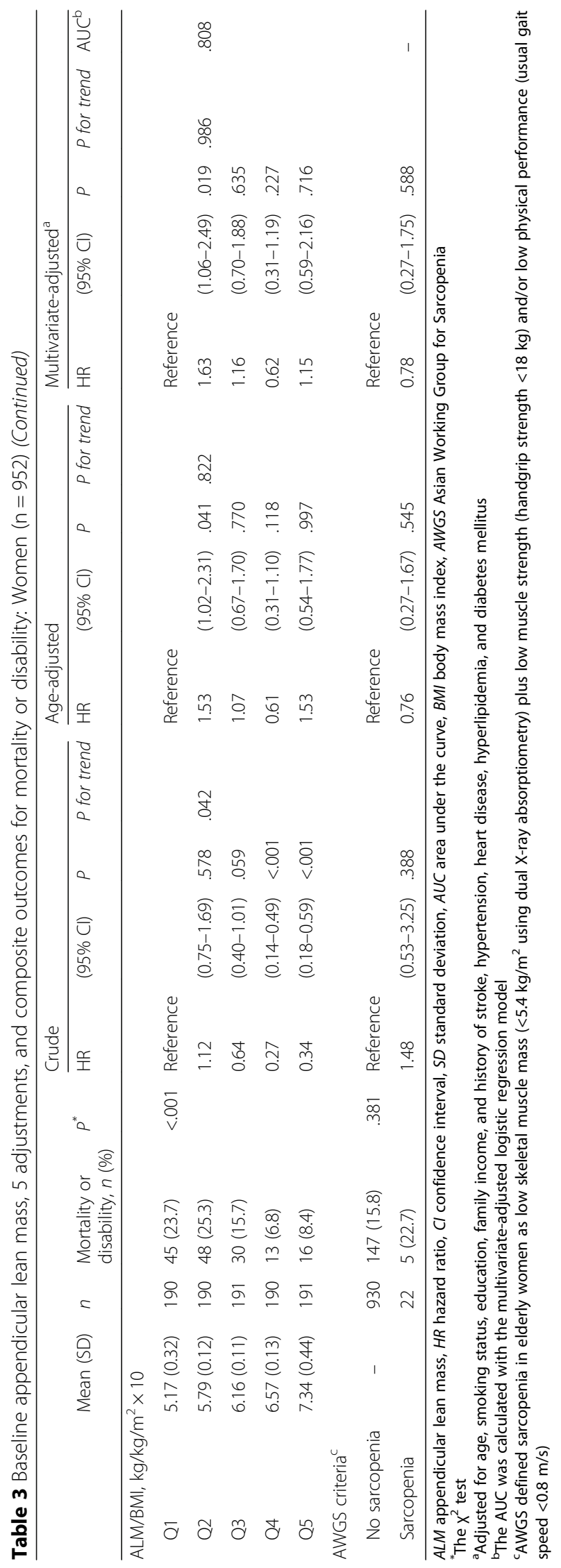


of ALM, ALM/leg length, ALM/height, and ALM/weight in women thus differed by age. The interaction of age and time was negative $(\beta<0, P<.001)$ for each ALM index in men and for ALM, ALM/leg length, and ALM/height in women, and the interaction was positive $(\beta>0, P<.05)$ only for ALM/weight in women. Therefore, the slope of each ALM index in men and ALM, ALM/leg length, and ALM/height in women differed by age at baseline. When we adjusted lifestyle-related factors including smoking status, alcohol intake, total physical activity, and energy intake, the association between age and muscle mass decline remained positive (data not shown). This finding means the indexes of decline in age-related muscle mass were independent of these lifestyle-related factors.

When we estimated the slope of ALM and the 5 ALM adjustments according to age at baseline, each ALM index among men started to decrease at 49 years old for ALM (49-year slope, $-0.012 \mathrm{~kg} /$ year, $P=.013$, Additional file 3 : Figure S1A), at 60 years old for ALM/leg length (60-year slope, $-0.017 \mathrm{~kg} / \mathrm{m} /$ year, $P=.003$, Additional file 3 : Figure S1B), at 50 years old for ALM/height (50-year slope, $-0.006 \mathrm{~kg} / \mathrm{m} /$ year, $P=.033$, Additional file 3 : Figure S1C), at 52 years old for ALM/height ${ }^{2}$ (52-year slope, $-0.004 \mathrm{~kg} / \mathrm{m}^{2} /$ year, $P=.017$, Additional file 3 : Figure S1D), at 50 years old for ALM/weight (50-year slope, $-0.013 \mathrm{~kg} / \mathrm{kg} \times 100 /$ year, $P=.038$, Additional file 3: Figure S1E), and at 45 years old for ALM/BMI (45-year slope, $-0.005 \mathrm{~kg} / \mathrm{kg} / \mathrm{m}^{2} \times 10 /$ year, $P=.025$, Additional file 3: Figure S1F).

For women, 3 ALM indices-ALM, ALM/leg length, and ALM/height-started to decrease at 40 years old for ALM (40-year slope, $-0.019 \mathrm{~kg} /$ year, $P<.001$, Additional file 3: Figure S1A), at 45 years old for ALM/leg length (45-year slope, $-0.014 \mathrm{~kg} / \mathrm{m} /$ year, $P=.029$, Additional file 3: Figure S1B), and at 40 years old for ALM/height (40-year slope, $-0.011 \mathrm{~kg} / \mathrm{m} /$ year, $P=.002$, Additional file 3: Figure S1C).

In the results summary, Crude Cox proportional hazard models and AUC indicated that higher ALM and ALM/BMI in women, and higher ALM, ALM/leg length, ALM/height, and ALM/BMI in men were associated with lower risks for mortality or disability than ALM/ height ${ }^{2}$. The higher coincidence rates of the lowest quintile among each ALM index were shown between ALM and ALM/leg length, ALM/height indexes, or ALM/leg length and ALM/height indexes, or ALM/height and ALM/height ${ }^{2}$ indexes in men and women, respectively. In addition, additional analyses using the mixed effect model indicated all ALM indexes in men, and ALM, ALM/leg length, and ALM/height in women could better predict age-related lean muscle mass decline. Considering the age-related muscle mass decline, unadjusted ALM would be the first variable to assess, regardless of sex, in this Japanese cohort study.

\section{Discussion}

This study indicated that crude ALM and ALM/BMI values in women and crude ALM, ALM/leg length, ALM/height, and ALM/BMI in men were positively associated with lower risks for mortality or disability, respectively. Data in the mixed effect model showed that interactions of age and time were negative for each ALM index in men $(\beta$ for age $\times$ time $<0, P<.001)$ and for ALM, ALM/leg length, and ALM/height in women ( $\beta$ for age $\times$ time $<0, P<.05$ ). The decreasing trend in these indices was greater among the elderly. Considering all results, including those in the mixed effect model, crude ALM for both sexes and ALM/leg length or ALM/height for men only appear more appropriate for predicting future mortality or disability compared with ALM/height ${ }^{2}$, which is currently used in the AWGS definition. Thus no adjustment for ALM to predict mortality or disability, regardless of sex, would be the best assessment, as it could reflect age-related muscle mass decline in both sexes.

Previous cross-sectional studies among Japanese subjects have indicated that the percentage of total skeletal muscle mass index with height adjustment decreased by $10.8 \%$ in men and by $6.4 \%$ in women among those aged 40 to 79 years [21], and that lean body mass divided by height ${ }^{2}$ and appendicular muscle mass divided by height ${ }^{2}$ were associated with grip strength [22]. Cross-sectional analysis showed that skeletal muscle mass index decreased with age only in men in the same Japanese cohort as this study [10]. In contrast, for women, SMI with heightadjustment (ALM/height $\left.{ }^{2}\right)$ was shown to represent a poor predictor of muscle mass decline among Chinese [13], Korean [9], and Japanese subjects [10]. The major reasons for sex differences in previous reports have been considered to be based on differences in body mass, body fat mass, hormones, and daily physical activity between men and women.

In terms of body fat, some studies have indicated that age-related fat mass [23, 24], height and fat mass, and BMI are all better for predicting disability $[25,26]$ or the prevalence of sarcopenia [27] than the ALM/height ${ }^{2}$ method [28]. In the present study, higher ALM/BMI was associated with lower risks for mortality or disability in both sexes, but the effect of the interaction of age and time on ALM/BMI was not significant in women. This means ALM/BMI does not decline with age in women; in other words, the index did not work well to predict age-related muscle mass decline in our cohort study.

In addition, ALM/weight did not predict future mortality or disability and did not show any decreasing trend with age. Thinness in young women represents a serious health concern in Japan [29], and age differences in weight or BMI may be seriously affected by cohort differences. In subanalyses, we examined associations between ALM-adjusted 
fat mass (\%) as measured by DXA, and mortality or disability, but found no significant associations between these variables in women (data not shown). However, men with a higher ALM/body fat showed lower risks for mortality or disability (hazard ratio [95\% confidence interval], Q1; reference, Q2; 0.80 [0.57-1.13], Q3; 0.57 [0.39-0.83], Q4; 0.43 [0.28-0.64], Q4; 0.36 [0.23-0.54], $P$ for trend <.001). Because body mass and body fat are generally lower in Japanese subjects than in Caucasians [30], adjusting for fat mass may be less useful when evaluating the age-related muscle mass decline among relatively lean ethnic groups.

In this study, we first considered leg length for ALM adjustment. One reason for the poor association between age and ALM/height ${ }^{2}$ among women was thought to be that adjustment using height ${ }^{2}$ may result in over-adjustment for the elderly. We have previously reported a longitudinal decreasing trend in height according to baseline age in the same cohort used in this study (age, 40-79 years) [31]. Loss of height began in men at 41 years and in women at 42 years, and the slope of height was -0.01 to $-0.17 \mathrm{~cm} /$ year among men $41-79$ years and -0.02 to $-0.25 \mathrm{~cm} /$ year among women $42-79$ years [31]. This means that the agerelated decreasing trend in height was greater in women than in men, and that height ${ }^{2}$ adjustment may result in an over-adjustment for women.

Several limitations must be considered when interpreting the results of this study. First, we assessed ALM using DXA measurements. Although DXA is one of the best ways to assess muscle mass, infiltration of fat into the muscle is difficult to distinguish [10, 32]. We compared the ALM adjustment of weight or BMI in this study, and these variables might be a better predictor of fat mass than DXA measurements. Reproducibility using different modalities such as computed tomography or bioelectrical impedance needs to be examined in future studies. Second, age- or multivariate-adjusted hazard ratios of ALM indices for disability or mortality were not statistically significant, but age should be a strong predictor of these outcomes. Additional analyses using the mixed effect model indicated all ALM indexes in men, and ALM, ALM/leg length, and ALM/height in women could better predict age-related lean mass decline. When we adjusted for confounding lifestyle-related factors, including smoking status, alcohol intake, total physical activity, and energy intake, the association for age-related muscle mass decline remained; therefore, non-adjusted ALM index could better predict age-related muscle mass decline both in men and women.

The main strengths of the present study are as follows: 1) the longitudinal design of our analyses lends strength to our inferences, as each individual was followed for more than 15 years, providing evidence of a causal association between ALM indices and disability; 2) use of a middle- and older-aged sample of randomly selected age- and sex-stratified non-institutionalized individuals from the community means that our results may be applicable to non-institutionalized Japanese elderly individuals; and 3) this is the first study to assess multiple ALM indices concurrently.

\section{Conclusions}

Longitudinal data showed that crude ALM for both sexes and ALM/leg length, ALM/height, or ALM/BMI for men are more appropriate to predict future disability compared to ALM/height ${ }^{2}$, which is currently used in the AWGS. Considering the age-related muscle mass decline, unadjusted ALM would be the first variable to assess, regardless of sex, in this Japanese cohort study.

Further studies are needed to support reproducibility of these results concerning the most appropriate methods for measuring muscle mass in Asian women.

\section{Additional files}

Additional file 1: Table S1. Coincidence rate of the lowest quintile among each variable (XLSX $10 \mathrm{~kb}$ )

Additional file 2: Table S2. Mixed-effects analyses of fixed effects for ALM and 5 adjustments of ALM over 12 years* (XLSX 27 kb)

Additional file 3: Figure S1. A. Estimated linear changes in ALM over 12 years by 4 -year age groups at baseline. B. Estimated linear changes in ALM/leg length over 12 years by 4-year age groups at baseline. C. Estimated linear changes in ALM/height over 12 years by 4 -year age groups at baseline. D. Estimated linear changes in ALM/height ${ }^{2}$ over 12 years by 4-year age groups at baseline. E. Estimated linear changes in ALM/weight over 12 years by 4-year age groups at baseline. F. Estimated linear changes in ALM/BMl over 12 years by 4-year age groups at baseline. (XLSX $181 \mathrm{~kb}$ )

\section{Acknowledgements}

We wish to express our sincere appreciation to the study participants and our colleagues in the NILS-LSA for completing the survey for this study.

\section{Funding}

This work was supported in part by grants from the Japanese Ministry of Education, Culture, Sports, Science and Technology (grant numbers 16H03264, $15 \mathrm{~K} 19253$ and 26293153) and by research funding from Longevity Sciences from the National Center for Geriatrics and Gerontology (grant number 28-40), Japan.

\section{Availability of data and materials}

The datasets generated and analyses performed during the current study are not publicly available due to the consent requirement of participants, but sexand age decade-stratified descriptive data are available from the corresponding author on reasonable request.

\section{Authors' contributions}

HS and FA designed the NILS-LSA first to seventh wave study. RO, HS, and FA designed the NILS-LSA follow-up survey in 2013. RO, YM, CT, YN, MT, FA, and HS substantially contributed to the collection of anthropometric and medical data. YN and MT created the postal survey and interpreted the data. HS, RO, and $C T$ applied and linked data to the National Vital Statistics records. HA and YM created the original research question. RO completed the statistical analysis with support from YN (mixed effect analyses) and CT (Cox proportional analyses). All authors interpreted the data. $\mathrm{RO}$ was a major contributor in writing the manuscript, and the other authors discussed and revised it. All authors approved the final version of the manuscript. 


\section{Ethics approval and consent to participate}

The study protocol was reviewed and approved by the Committee of Ethics of Human Research at the National Center for Geriatrics and Gerontology (no. 899). Written informed consent was obtained from all subjects during the first to the seventh waves of the NILS-LSA. In the survey mailed in 2013, we explained that returning the self-administered questionnaire represented informed consent

\section{Consent for publication}

Not applicable

\section{Competing interests}

The authors declare that they have no competing interests.

\section{Publisher's Note}

Springer Nature remains neutral with regard to jurisdictional claims in published maps and institutional affiliations.

\begin{abstract}
Author details
'Section of NILS-LSA (National Institute for Longevity Sciences-Longitudinal Study of Aging), Center for Gerontology and Social Science, National Center for Geriatrics and Gerontology, 7-430 Morioka-cho, Obu, Aichi 474-8511, Japan. ${ }^{2}$ Department of Orthopedics, National Center for Geriatrics and Gerontology, 7-430 Morioka-cho, Obu, Aichi 474-8511, Japan. ${ }^{3}$ Faculty of Health and Medical Sciences, Aichi Shukutoku University, 2-9 Katahira, Nagakute, Aichi 480-1197, Japan. ${ }^{4}$ Graduate School of Nutritional Sciences, Nagoya University of Arts and Sciences, 57 Takeyanoyama, Iwasaki-cho, Nisshin, Aichi 470-0196, Japan. ${ }^{5}$ Hospital, National Center for Geriatrics and Gerontology, 7-430 Morioka-cho, Obu, Aichi 474-8511, Japan.
\end{abstract}

Received: 31 July 2017 Accepted: 21 December 2017

Published online: 05 January 2018

\section{References}

1. Rosenberg I. Epidemiologic and methodologic problems in determining nutritional status of older persons. Am J Clin Nutr. 1989;50(5 Suppl):1121-235.

2. Baumgartner RN, Koehler KM, Gallagher D, et al. Epidemiology of sarcopenia among the elderly in New Mexico. Am J Epidemiol. 1998;147:755-63.

3. Morley JE. Sarcopenia in the elderly. Fam Pract. 2012;29:144-8.

4. Arai H, Akishita M, Chen LK. Growing research on sarcopenia in Asia. Geriatr Gerontol Int. 2014;14(Suppl 1):1-7.

5. Chang SF, Lin PL. Systematic literature review and meta-analysis of the association of sarcopenia with mortality. Worldviews Evidence-Based Nurs. 2016;13:153-62.

6. Chen LK, Liu LK, Woo J, et al. Sarcopenia in Asia: consensus report of the Asian working group for sarcopenia. J Am Med Dir Assoc. 2014;15:95-101.

7. Cruz-Jentoft AJ, Baeyens JP, Bauer JM, et al. Sarcopenia: European consensus on definition and diagnosis: report of the European working group on sarcopenia in older people. Ageing. 2010;39:412-23.

8. Kim YP, Joh JY, Kim S, et al. The application of different appendicular skeletal muscle cutoff points and research definitions associated with health-related quality of life in Korean older people: data from KNHANES 2008-2011. BMC Geriatr. 2014;14:144.

9. Kim YS, Lee Y, Chung YS, et al. Prevalence of sarcopenia and sarcopenic obesity in the Korean population based on the fourth Korean National Health and nutritional examination surveys. J Gerontol A Biol Sci Med Sci. 2012:67:1107-13.

10. Shimokata $H$, Ando F, Yuki A, et al. Age-related changes in skeletal muscle mass among community-dwelling Japanese: a 12-year longitudinal study. Geriatr Gerontol Int. 2014;14(Suppl 1):85-92.

11. Studenski SA, Peters KW, Alley DE, et al. The FNIH sarcopenia project: rationale, study description, conference recommendations, and final estimates. J Gerontol A Biol Sci Med Sci. 2014;15: 69(5):547-558.

12. Moon JH, Kim KM, Kim JH, et al. Predictive values of the new sarcopenia index by the Foundation for the National Institutes of Health Sarcopenia Project for Mortality among older Korean adults. PloS one. 2016;11(11): e0166344

13. Wen $X$, Wang $M$, Jiang $C M$, et al. Are current definitions of sarcopenia applicable for older Chinese adults? J Nutr Health Aging. 2011;15:847-51.
14. Cawthon PM, Peters KW, Shardell MD, et al. Cutpoints for low appendicular lean mass that identify older adults with clinically significant weakness. J Gerontol A Biol Sci Med Sci. 2014;69(5):567-75.

15. Reijnierse EM, Trappenburg MC, Leter MJ, et al. The impact of different diagnostic criteria on the prevalence of sarcopenia in healthy elderly participants and geriatric outpatients. Gerontology. 2015;61(6):491-6.

16. Spira D, Buchmann N, Nikolov J, et al. Association of low lean mass with frailty and physical performance: a comparison between two operational definitions of sarcopenia-data from the Berlin aging study II (BASE-II). J Gerontol A Biol Sci Med Sci. 2015;70(6):779-84

17. Shimokata $\mathrm{H}$, Ando F, Niino N. A new comprehensive study on aging-the National Institute for longevity sciences, longitudinal study of aging (Nils-LSA). J Epidemiol. 2000;10:S1-9.

18. Campbell JC, Ikegami N. Long-term care insurance comes to Japan. Health Aff. 2000;19:26-39.

19. Monograph. "Follow-Up Postal Survey 1" of the first wave participants Date of implementation: August 2013. 2013. http://www.ncgg.go.jp/cgss/ department/ep/survey/index.html Accessed 15 Dec 2017.

20. Monograph.The First Wave, November, 1997 - April, 2000, NILS-LSA. 2000. http://www.ncgg.go.jp/cgss/department/ep/monograph1st/index.html Accessed 15 Dec 2017.

21. Yamada M, Nishiguchi S, Fukutani $N$, et al. Prevalence of sarcopenia in community-dwelling Japanese older adults. J Am Med Dir Assoc. 2013;14:911-5.

22. Liu LK, Lee WJ, Liu CL, et al. Age-related skeletal muscle mass loss and physical performance in Taiwan: implications to diagnostic strategy of sarcopenia in Asia. Geriatr Gerontol Int. 2013;13:964-71.

23. Estrada $\mathrm{M}$, Kleppinger $\mathrm{A}$, Judge $\mathrm{JO}$, et al. Functional impact of relative versus absolute sarcopenia in healthy older women. J Am Geriatr Soc. 2007;55:1712-9.

24. Dufour AB, Hannan MT, Murabito JM, et al. Sarcopenia definitions considering body size and fat mass are associated with mobility limitations: the Framingham study. J Gerontol A Biol Sci Med Sci. 2013;68:168-74.

25. Delmonico MJ, Harris TB, Lee JS, et al. Alternative definitions of sarcopenia, lower extremity performance, and functional impairment with aging in older men and women. J Am Geriatr Soc. 2007;55:769-74.

26. McLean RR, Shardell MD, Alley DE, et al. Criteria for clinically relevant weakness and low lean mass and their longitudinal association with incident mobility impairment and mortality: the foundation for the National Institutes of Health (FNIH) sarcopenia project. J Gerontol A Biol Sci Med Sci. 2014:69:576-83.

27. Newman AB, Kupelian V, Visser M, et al. Sarcopenia: alternative definitions and associations with lower extremity function. J Am Geriatr Soc. 2003;51:1602-9.

28. Morley JE, Abbatecola AM, Argiles JM, et al. Sarcopenia with limited mobility: an international consensus. J Am Med Dir Assoc. 2011;12:403-9.

29. Takimoto H, Yoshiike N, Kaneda F, et al. Thinness among young Japanese women. Am J Public Health. 2004;94:1592-5

30. Deurenberg P, Yap M, van Staveren WA. Body mass index and percent body fat: a meta analysis among different ethnic groups. Int J Obes Relat Metab Disord. 1998:22:1164-71.

31. Otsuka R, Kato $Y$, Nishita $Y$, et al. Age-related changes in energy intake and weight in community-dwelling middle-aged and elderly Japanese. J Nutr Health Aging. 2016;20:383-90.

32. Kasai T, Ishiguro N, Matsui $Y$, et al. Sex- and age-related differences in midthigh composition and muscle quality determined by computed tomography in middle-aged and elderly Japanese. Geriatr Gerontol Int. 2015;15:700-6.

\section{Submit your next manuscript to BioMed Central and we will help you at every step:}

- We accept pre-submission inquiries

- Our selector tool helps you to find the most relevant journal

- We provide round the clock customer support

- Convenient online submission

- Thorough peer review

- Inclusion in PubMed and all major indexing services

- Maximum visibility for your research

Submit your manuscript at www.biomedcentral.com/submit
Biomed Central 